See discussions, stats, and author profiles for this publication at: https://www.researchgate.net/publication/336728471

\title{
PRYSTINE - Technical Progress After Year 1
}

Conference Paper · August 2019

DOI: 10.1109/DSD.2019.00063

\section{CITATIONS}

0

23 authors, including:

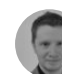

Norbert Druml

Infineon Technologies

73 PUBLICATIONS 172 CITATIONS

SEE PROFILE

Georg Macher

AVL LIST GMBH

66 PUBLICATIONS 297 CITATIONS

SEE PROFILE
READS

40

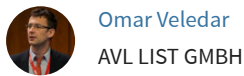

17 PUBlications 422 CITATIONS

SEE PROFILE

S. Solmaz

Virtual Vehicle

61 PUBLICATIONS 393 CITATIONS

SEE PROFILE

Some of the authors of this publication are also working on these related projects:

PRYSTINE View project

Project $\quad$ ADAS - Advanced Driver Assistance Systems View project 


\section{PRYSTINE - Technical Progress After Year 1}

Norbert Druml*, Omar Veledar, Georg Macher, Georg Stettinger, Solmaz Selim, Jakob Reckenzaun, Sergio E. Diaz, Mauricio Marcano, Jorge Villagra, Rutger Beekelaar, Johannes Jany-Luig, Marta Maria Corredoira Sagues, Paolo Burgio, Christian Ballato, Björn Debaillie, Lars van Meurs, Andrei Terechko, Fabio Tango, Anna Ryabokon, Andrei Anghel, Oğuz İçoğlu, Sumeet S. Kumar, George Dimitrakopoulos

\section{*Infineon Technologies Austria AG, Austria, norbert.druml@infineon.com}

\begin{abstract}
Among the actual trends that will affect society in the coming years, autonomous driving stands out as having the potential to disruptively change the automotive industry as we know it today. For this, fail-operational behavior is essential in the sense, plan, and act stages of the automation chain in order to handle safety-critical situations by its own, which currently is not reached with state-of-the-art approaches also due to missing reliable environment perception and sensor fusion.

PRYSTINE will realize Fail-operational Urban Surround perceptION (FUSION) which is based on robust Radar and LiDAR sensor fusion and control functions in order to enable safe automated driving in urban and rural environments. In this paper, we detail the vision of the PRYSTINE project and we showcase the results achieved during the first year.
\end{abstract}

Keywords-FUSION, fail-operational, perception

\section{INTRODUCTION}

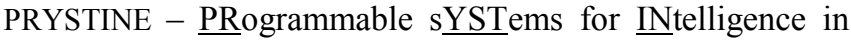
AutomobilEs - implements a matrix-style organization made of work packages and supply chains. A supply chain is the logical / virtual combination of partner activities fitting together within a specific topic leading to a combined result (e.g., demonstrator). A supply chain supplies other supply chains and project activities with its results. Each supply chain addresses a specific PRYSTINE objective. PRYSTINE's objectives can be distinguished between technical objectives (O1-O4) and impact/social objectives (O5-O6). Thus, a supply chain's result (demonstrator) addresses the specific objective (for example, the sensor components developed in supply chain 1 will demonstrate enhanced reliability and performance, reduced cost and power). Every effort / activity of a specific partner in a supply chain is mapped into the generic work packages, starting with requirements, and ending with validation and test (which represents the typical automotive V-development cycle). This approach forms a clearly arranged matrix structure, which is depicted in Fig. 1. PRYSTINE defines three types of supply chains.

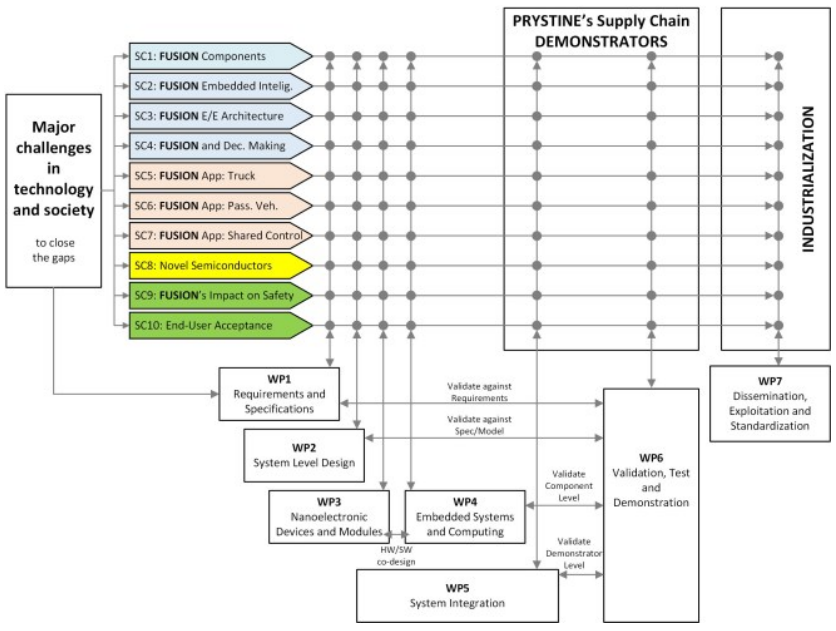

Fig. 1: PRYSTINE's WP and SC interaction reflecting the automotive Vdevelopment cycle. Obtained with changes from [1].

The technology enabler supply chains (1-4) develop the fundamental core technology bricks required by other supply chains and for achieving PRYSTINE's ambitious goals. These supply chains 1-4 cover fail-operational semiconductor components, embedded systems and fundamental algorithms, E/E vehicle architecture as well as the important sensor fusion solutions, thus realizing FUSION.

The output enabler (also known as applications or shiny demonstrators) supply chains (5-7) employ and validate the results achieved in the technology enabler supply chains. Therefore, the advancements achieved during the PRYSTINE project will be showcased by dedicated demonstrators of supply chains $5-7$. In particular, a heavy-duty vehicle demonstrator, a passenger vehicle demonstrator, and a shared control demonstrator will be featured.

The impact supply chains (8-10) form the basis in order to generate "European Values". This includes economic, societal, and panEuropean impact factors generated by the PRYSTINE project.

\section{PRYSTINE'S SUPPLY CHAINS}

\section{SC1: Components LiDAR, Radar and safety controllers for} FUSION

$\mathrm{SC} 1$ represents one of the core technology enablers. The vision of this supply chain and its project partners is to research and develop PRYSTINE's essential components for FUSION. Particularly important is the focus on fail-operational environment perception sensors (Radar \& LiDAR) and on next-generation embedded control components (such as safety controllers and processing hardware). These core components will be employed and integrated by most of PRYSTINE's supply chains. Furthermore, SC1 will address the following measureable key performance indicators:

- Fail-operational sensor compound vs. fail silent individual sensing approaches

- Power reduction of $25 \%$ through semiconductor material improvements and functional convergence in sensor modules

- Up to $30 \%$ cost reduction and $10 \%$ margin improvement for perception sub-systems

- $\quad 30 \%$ less false-positive and/or false-negative detections compared to separate sensing approach

SC1 pursues two approaches towards fail-operational LiDAR sensing: an oscillating comp-drive based 1D MEMS scanning solution and a piezo electric 2D MEMS scanning approach. Both approaches come with their distinct pros and cons. For example, while 1D scanners typically enable high frame rates and show high robustness against vibrations and shocks, 2D scanners (see also Fig. 2) concentrate the whole laser light into a single laser spot thus enabling high SNR values and long-range scanning.
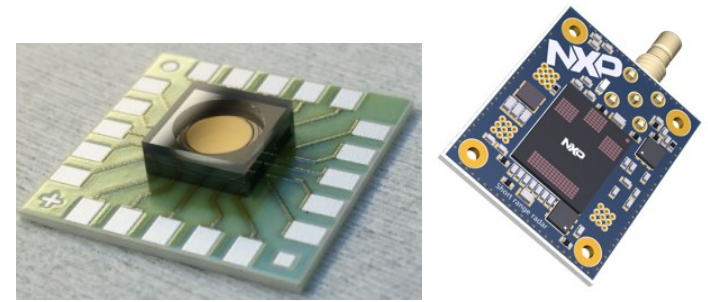

Fig. 2: Murata's 2D scanning MEMS mirror and NXP's Radar module. 
Radar is considered as one of the key sensors for automated driving because it provides reliable detection over a wide range for weather conditions (rain, snow, lighting, fog, etc.). The recent trend of designing Radars in CMOS technologies comes with several additional advantages. CMOS is a low-cost technology which supports dense integration, and will continue to scale-down towards future nodes. This is a clear benefit for automotive, as large-scale deployment and seamless integration is targeted. At the same time, CMOS supports complex system integration allowing to integrate both the RF sensor and the digital data processing on the same chip. In terms of cost scaling and miniaturization, CMOS clearly outperforms cameras and other sensors. Therefore, CMOS Radars development is one of the key activities in PRYSTINE. For example, a novel package is developed that integrates not only a CMOS transceiver but also MIMO antennas, thus forming an innovative System-in-Package, see also Fig. 2.

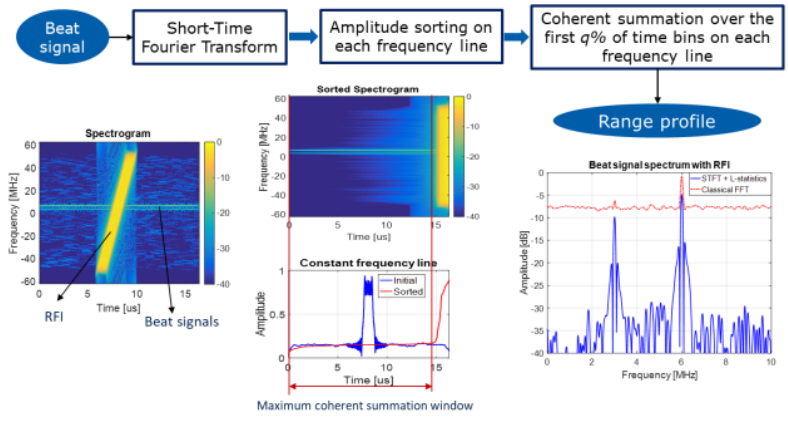

Fig. 3: UPB's single Radar RFI mitigation algorithm (block diagram and preliminary results).

From the Radar signal processing point of view, the efforts were concentrated during the first year on distributed Radar sensors, direction of arrival estimation methods and radio frequency interference (RFI) detection and mitigation algorithms. For instance, a single Radar RFI mitigation algorithm based on short time Fourier transform and a linear combination of order statistics (L-statistics) was designed (a general block diagram of the algorithm and preliminary results on simulated data are shown in Fig. 3). In the presence of a typical Radar-to-Radar interference, the algorithm retains most of the target's energy in the computed range profile, which makes it suitable for an interference-operational Radar sensor.

In addition to the aspects mentioned above, IMEC targets their Radar sensor as a building block for a fail-operational system. The envisioned Radar sensor will offer scalability over its sensitivity versus its power consumption. Having such scalability enables the Radar sensor to maintain its operation on an emergency cell battery in case of a central power breakdown. In that case, the remaining battery power would be reserved for driving the power train, and the reduced sensing sensitivity would be sufficient for the low vehicle speed. On the other hand, the Radar sensor could be scaled to maximal sensitivity (and power consumption) to take-over the functionality of other failing sensors. In addition to scalability, IMEC develops their Radar sensor for high robustness and reliability to minimize false detections and interferences.

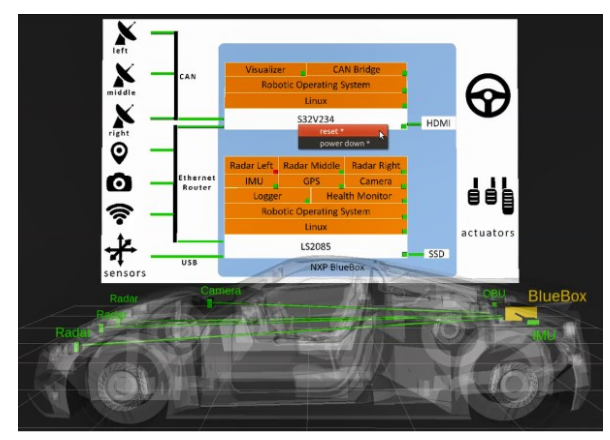

Fig. 4: Vehicular health monitoring solution, obtained from [1].
SC1 also spends lots of efforts in order to advance current safety controllers and health monitoring solutions way beyond state-ofthe-art. This is of particular importance, because these safety components form the safety-backbone of current and future vehicles. Fig. 4 depicts an innovative solution towards a vehicular health monitoring solution. The vehicle's hardware and software is continuously monitored. In case a fault is detected, alarms are triggered and certain safe states are entered that, e.g., continue to operate the vehicle in a degraded mode.

SC2: High performance embedded control and intelligence for FUSION

$\mathrm{SC} 2$ is the second core technology enabler that addresses Objective 2: Dependable embedded control by co-integration of signal processing and AI approaches for FUSION on the system level by realizing highly-efficient dependable architectures. These novel architectures should fulfill crucial functional safety requirements for automated driving and ADAS functions based on sensor data fusion and include two main architectures of R\&D:

1. Embedded Control architectures by combining COTS components, e.g. number crunching processors, safety microcontrollers, Deterministic Ethernet backbone for data exchange, and

2. Embedded Intelligence architectures such as "drive-bywire" cars, AI algorithms for self- and context-awareness for the road safety and system security.

The developments within SC2 are one of the most important prerequisites to realize the fail-operational automated driving. The main activities in SC2 include collection of requirements as well as the design, development, and proof-of-concept demonstration of a computing infrastructure. The main goal of these developments is to support various control mechanisms that must remain fully/partially functional in case of faults or impairments in the environment perception. The future architectures will implement redundancy and diversity within the electronic control units using highly-performant and cost-efficient computational components. There are four subobjectives defined in $\mathrm{SC} 2$ to achieve $\mathrm{O} 2$ :

O2.1: Fail-operational automated driving platform (SAE Level 3+) exploiting COTS components and Deterministic Ethernet backbone network to enable safety-critical application data exchange

O2.2: Design of architectures by component diversification to overcome ability to fail by achieving each system independent functionality with certain level of robustness and development of Automated Safety and Awareness Processing Stack

O2.3: Develop solutions for monitoring vehicle internal communications for intrusions and for controlling vehicle internal and external network boundary; develop a trust model for reliability of internal data, sensors and system state as well a driver monitoring subsystem to guarantee a successful precision traffic movement.

O2.4: Development of SAE Level a 3+ equivalent autonomous parking solution and prototype FUSION algorithms for low speed autonomy based on Virtual Vehicle passenger vehicle pre-demonstrator (described below) to be integrated in Ford heavy duty truck demonstrator for low speed autonomy.

The expertise of SC2 partners fits perfectly to the mentioned objectives and covers, for instance, the next generation HW such as networking and safety controllers, real-time software and corresponding interfaces, AI approaches including heuristic search and machine learning, build-in security mechanisms, etc. These developments are implemented in five tasks and four demonstrators, which exactly corresponds to the sub-objectives defined above.

One of the demonstrators in this supply chain focuses on the low speed autonomy and automated back-parking use case. This pre- 
demonstrator is based on a passenger vehicle and it also contributes to the demonstrators in SC4 and SC5. The FUSION algorithms will be developed in a co-simulation environment, which serve as a basis to simulate the individual use-case scenarios and to verify the designed FUSION algorithms virtually, see Fig. 5.

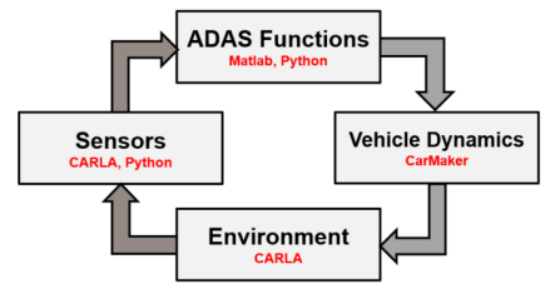

Fig. 5: Co-simulation environment structure.

The environment simulations will be based in open source CARLA platform, while the vehicle dynamics model will be simulated using the IPG Carmaker/TruckMaker. The ADAS control functions will be based on Matlab/Python whereas sensor models will be modelled in Python/Carla.

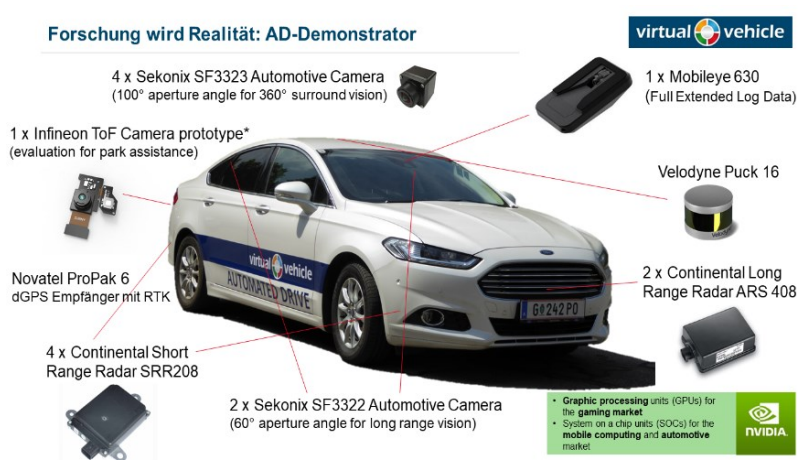

Fig. 6: Demonstrator vehicle used on the low-speed autonomy use cases.

The test vehicle to be used for the demonstration is prepared and provided by Virtual Vehicle with a range of sensors as seen in Fig. 6. In addition to the simulation environment development, a draft architecture for the perception components as well as the HW/SW interfaces and the $\mathrm{E} / \mathrm{E}$ architecture is being developed in the scope the specific use cases that are shown in Fig. 7.

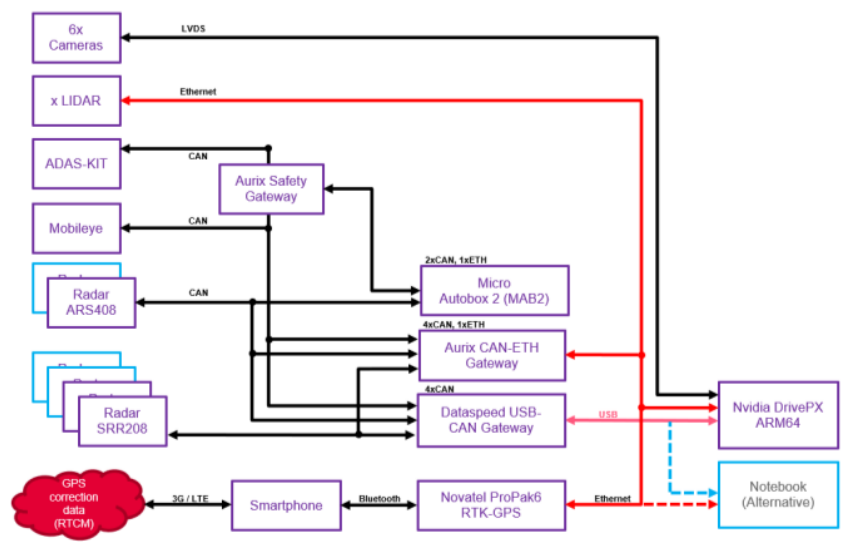

Fig. 7: $\mathrm{E} / \mathrm{E}$ and $\mathrm{HW} / \mathrm{SW}$ architecture of the pre-demonstrator.

The current status of the development process focusses on the individual perception layer components based on the co-simulation platform.

\section{SC3: Fail-operational E/E architecture enabling FUSION}

An automated vehicle must sense its environment, plan actions and react accordingly. An E/E system covers three aspects. As technology-enabler, SC 3 delivers a fail-operational E/E architecture of an E/E system. This E/E system connects the failoperational and optimized sensors, components, embedded safety controllers from other SCs using dependable vehicular electrical/electronic infrastructure and communication systems. The results will be shown in three demonstrators considering recent safety standardization activities.

The first demonstrator is an integration platform which supports the development of a generic fail-operational E/E system. The platform serves as basis to enhance and adapt architectures; and supports in partitioning functionality and control strategies. The overall aim of this platform is to enable an efficient integration of various FUSION technologies with a strong focus on dependability, testing and validation.

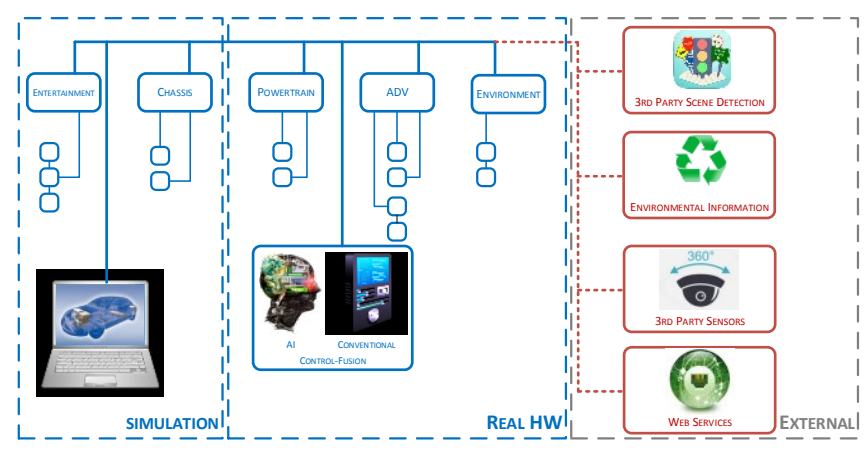

Fig. 8: SC 3 Demonstrator 1 \& 2, E/E-integration Plattform for E/E system Developlement.

The second demonstrator is closely linked to the previous one. It is a Framework for simulation, development and validation of novel AD functionalities that rely on distributed and highly interconnected control functions. The provided framework allows a seamless shift from pure simulation environment towards mixed physical and simulated environment to finally pure physical development for autonomous driving functionalities. It aims to interface between various hardware and software components in a FUSION environment and to enable their integration.

The third demonstrator is a dynamically shaped reliable mobile communication. It will be shown at a passenger vehicle, but also via a server. Its overall aim is to deliver an optimal connection for a vehicle via an optimal network. A degrading quality of service will trigger the underlying algorithm to choose a different network. The proposed process is shown in Fig. 9.

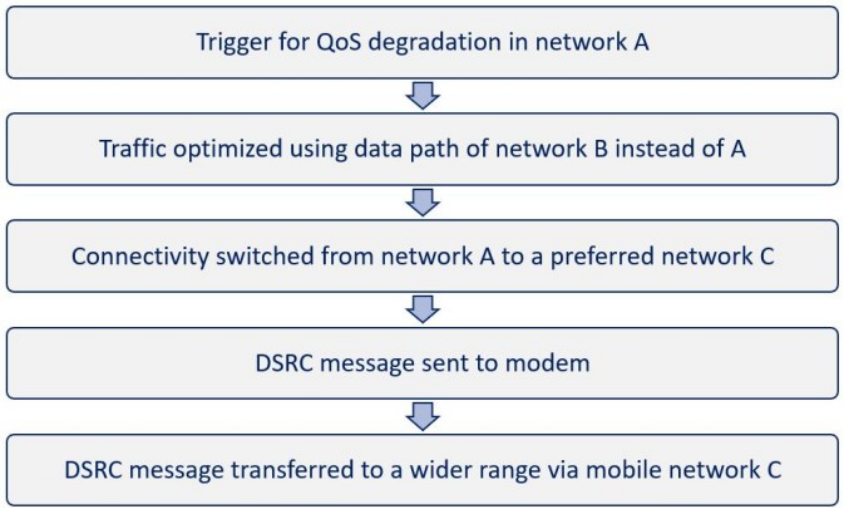

Fig. 9: Process for a QoS.

Advances in development of automated driving bears huge challenges to standardization processes and bodies. Various longestablished standards are ensuring a safe and secure $\mathrm{E} / \mathrm{E}$ architecture. The ISO 26262 "Road Vehicles - Functional Safety" (revised in 2018) must be applied when an E/E system bears the risk of potentially harming or killing a human being due to a malfunction of the system, its sub-systems and components or the 
interactions within the system. To ensure a safe operation, measures need to be taken, to reduce the risk of occurring to an acceptable minimum.

The ISO 26262 reduces hazardous events in a safety-related E/E system due to malfunction. For a highly automated vehicle, deviation of intended functionality can happen without any malfunctioning behavior of the system. Such vehicles need to handle level of uncertainty in a real road setting in order to achieve a proper situational awareness for a safe operation.
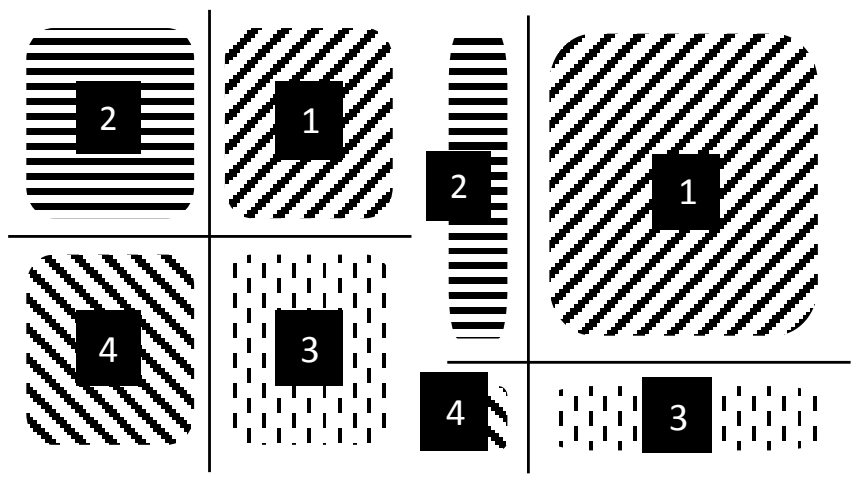

known \& safe scenarios

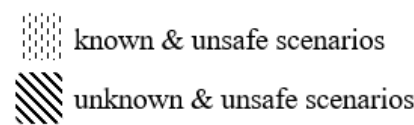

unknown \& safe scenarios

unknown \& unsafe scenarios

Fig. 10: Known and unknown scenarios.

The recently released ISO PAS 21448 "Safety of Intended Function" fills a white spot as it addresses automated vehicles that show an unexpected behavior, despite properly functioning elements in the $\mathrm{E} / \mathrm{E}$ system. An approach to tackle and to reduce such a behavior is discussed in the ISO PAS using a classification scheme of potential scenarios. The authors distinguish two different characteristics of a scenario. They argue that there are known and unknown as well as safe and unsafe scenarios; a permutation leads to four areas, shown in Fig. 10 (left). The Aim of a SOTIF process is to maximize or maintain area 1 , minimize area 2 with technical measures and minimize area 4.

During this project, the ISO PAS 21448 will be applied for test purposes to suitable use cased and derived scenarios. Applicability will be evaluated and results will be reported it to the relevant standardization body.

\section{SC4: FUSION and decision making}

SC4 is the fourth core technology enabler supply chain that addresses the third and fourth objectives of PRYSTINE:

Objective 3 - Optimized E/E architecture enabling FUSIONbased automated vehicles: This objective is achieved by incorporating PRYSTINE's fail-operational and optimized sensors, components, embedded controllers, processing systems with dependable vehicular electrical/electronic infrastructure. Thus PRYSTINE's FUSION technology will be realized and a failoperational electrical/electronic reference architecture (consisting also of control strategies and actuators) will be achieved.

Objective 4 - Fail-operational systems for urban and rural environments based on FUSION: This objective is achieved by implementing PRYSTINE's fail-operational sensor fusion, content analysis, object recognition, scenario assessment, and decision making solutions on PRYSTINE's fail-operational embedded control and electrical/electronic architectures. Thus, fail-operational automated driving functions for urban and rural environments will be achieved, which will be showcased by PRYSTINE's use-cases.

SC4 is comprised of robust perception of the environment around a vehicle, through the fusion of sensed data from a multitude of sensors (Radar, LiDAR, cameras...), thus realizing PRYSTINE's FUSION technology. SC4's vision is to implement; (i) fail- operational perception based on sensor fusion and content analysis, object recognition, scenario assessment, motion estimation, decision making, and see through solutions.

SC4 has close ties with SC1, SC2 and SC5, SC6, SC7. SC4 aims to integrate sensors developed in $\mathrm{SC} 1$ and the fusion box developed in $\mathrm{SC} 2$ with robust environment perception and fusion algorithms developed within itself. The final demonstrator of SC4 is a FUSION Hardware In the Loop (HIL) demonstration (see Fig. 11), namely the demonstration of successful results of SC4 in a HIL test setup. Subsequently, the results validated in the HIL tests will be conveyed to output enabler supply chains (SC5, SC6, and SC7) that will consequently demonstrate FUSION outputs in a heavy duty truck, passenger car and shared control environment respectively. To sum up in short, FUSION components, such as sensors (LiDAR, Radar, camera...), safety controllers, and PRYSTINE's reference embedded intelligence will be integrated to a demonstration setup in the lab environment.

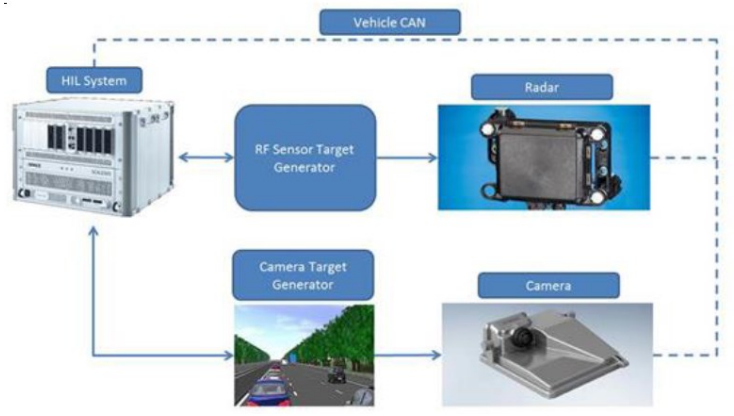

Fig. 11: A HIL environment with sensors and vehicle data employed.

First, simulators and emulators will be employed in the HIL setups. Perception and data fusion algorithms will be validated through simulated sensor data by being executed on emulated platforms. These simulators and emulators will further be replaced by the real hardware as the hardware arrives for integration. Thus, the sensor data will be recorded simultaneously with the vehicle data, then replayed for evaluation while the target platforms (hardware) are in the loop. These requirements explicitly address the key performance indicators of SC4; (i) synchronized recording of the multi sensor data and replay capability and (ii) fusion performance evaluation using recorded data from the vehicle. The FUSION HIL demonstrators will be divided into; (i) interfacing, (ii) functionality, (iii) performance and (iv) reliability testing sub-sections, and will be capable of testing the main functionality before installation on the heavy duty truck (SC5), passenger car (SC6) or shared control demonstrator (SC7).

SC4 involves 14 partners; Ford Otosan, AVL-Turkey, Virtual Vehicles, Unimore, Innoluce, Delft University of Technology, Imec, Dat.Mobility, Polito, Tampere University, Mattersoft, Nokia, Tenneco and ITI. The solutions implemented by the partners during the first year are given below:

a. LiDAR sensor data augmentation: LiDARs support very good range resolutions and provide good capabilities to classify objects. By fusing Radar, LiDAR, and camera well-balanced environment perception is achieved and challenging environmental conditions are mitigated. Innoluce supports the realization of the SC4 solutions with expertise in the fields of LiDAR system and application engineering in order to guarantee successful sensor integration. Towards this end in the scope of SC4, Innoluce develops sensor data augmentation algorithms to increase the quality of LiDAR sensor outputs (Fig 12). The LiDAR solution is utilized within SC4 by partners Ford and Tenecco for LiDAR based perception solutions, particularly "back maneuvering assist" and "suspension control" solutions respectively. The LiDAR efforts of Innoluce will eventually be transferred to SC5 "Heavy Duty Vehicle demonstration" and SC6 "Passenger Car demonstration" after successful HIL tests finalizing the SC4 studies. 

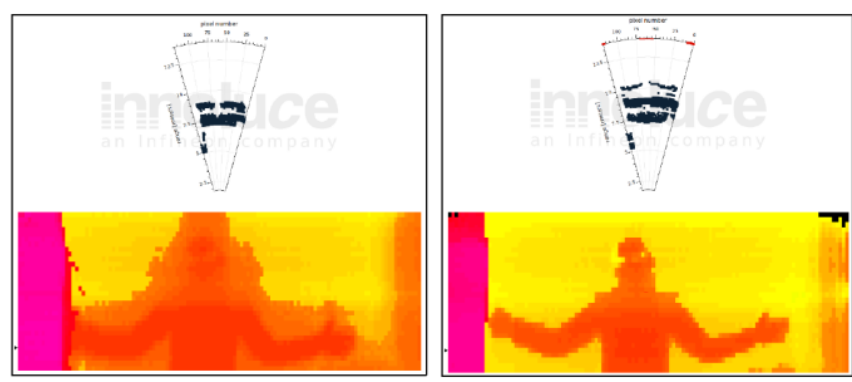

Fig 12: Examples of LiDAR sensor outputs: non-optimal outputs (left), optimal and augmented outputs (right).

b. Back Maneuvering Assist: Most autonomous developments today concentrate on long-haul highway related topics for heavyvehicles. The need for high-precision maneuvers, combined with the size of heavy-vehicles, raises a high fatality risk, threatening the accident-free mobility scenarios envisaged for Europe. Within the scope of SC4, back maneuvering assisting solutions are developed by Ford, AVLTR and VIF for articulated heavy duty vehicles. In the first year, perception algorithms are developed and objects are detected from LiDAR and stereo camera sensors by Ford. Offline open source test data are utilized for validation of the algorithms. Meanwhile simulated sensor data is being generated by AVLTR using Unreal Engine tool to be utilized for further perception testing (Fig. 13). Data Fusion algorithm is developed by AVLTR for fusing objects detected from various sources (LiDAR, stereo camera, Radar, etc.). Path planning algorithm is developed by Ford for further use to extract optimized paths for back maneuvering. These efforts will eventually be transferred to SC5 "Heavy Duty Vehicle demonstration" after successful HIL tests finalizing the SC4 studies.

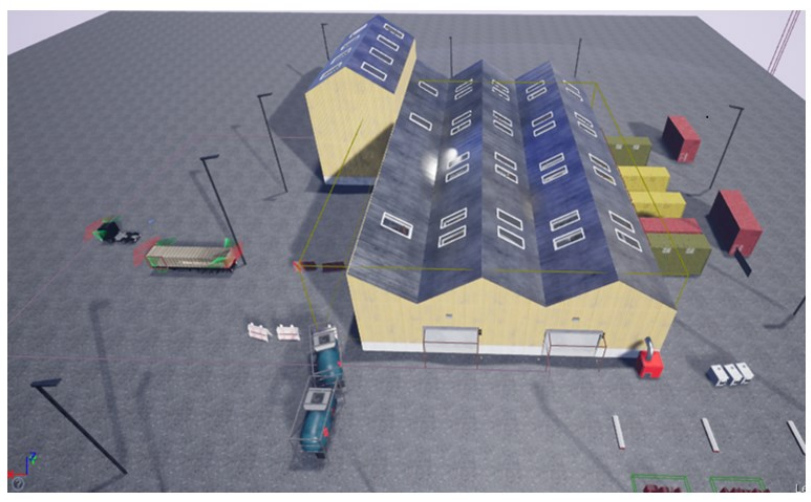

Fig. 13: Simulated parking station environment for back maneuvering assist developed with Unreal Engine.

c. Vulnerable Road User (VRU) Detection: In the EU, 22\% of road fatalities are pedestrians, while $8 \%$ are cyclists. Within PRYSTINE FUSION technologies, two core technologies dedicated to protecting these groups are developed. First one is the "vulnerable road user detection" towards VRU detection solutions, IMEC develops a platform for tracking and classification of VRUs based on raw data fusion of Radar and vision with algorithms offering higher robustness and fail-operational features. In the first year, the impact and means to mitigate Radar interference in a highly sensorized environment is investigated by IMEC and the findings are presented and published at RadarConf and IET conferences. IMEC's goal is to finally develop a reliable VRU detection/tracking/classification based on sensor fusion at a smart intersection (Fig. 14)

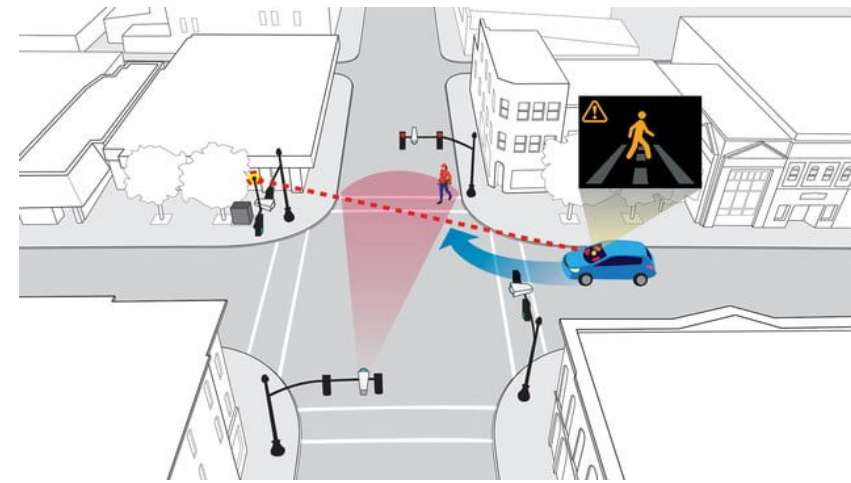

Fig. 14: Reliable VRU detection at a smart intersection.

Additionally, ITI developed deep learning based solutions for detection and recognition of VRUs using Single Shot Detector (SSD) and You Only Look Once (YOLO) methods, after observing different machine learning (ML) algorithms in terms of robustness and precision. In the same way, a subset of four databases (Caltech, Daimler, KITTI, BDD100k) from all the datasets were selected in different scenarios. An architectural specification of a computer vision framework is developed to generalize the common computer vision tasks. Currently, ITI is working in the development of the framework, and the first results are obtained by using it. Particularly, the framework is used to create, train and compare object detection ML algorithms with the above developed methods. These efforts of ITI will eventually be transferred to SC6 "Passenger Car demonstration" after successful HIL tests finalizing the $\mathrm{SC} 4$ studies.

d. SuperSight (CiThruS): As mentioned above, $22 \%$ of road fatalities are pedestrians and $8 \%$ are cyclists in the EU. Towards protecting these two groups, in addition to VRU detection, a second solution is developed in PRYSTINE; SuperSight, or in other words CiThruS is created with collaboration of TAU, MTS and Nokia. The goal of the SuperSight solution is to basically provide (i) blind area removal which is especially useful to spot weak road users before they enter the natural field of view. In addition SuperSight provides (ii) automatic safety alerts which reduce road accidents and improve driver proactivity, and (iii) traffic analytics which improves driving experience by helping the driver to bypass traffic jams, roadworks, and other unwanted events in the traffic. SuperSight solution utilizes 360 degrees video processing with surrounding cameras attached on the vehicle. In the first year, TAU focused on designing augmented 360 degrees spherical video view for the CiThruS framework. The addressed components of the realtime 360 degrees video processing pipeline include video capture, stitching, projection, HEVC (high efficiency video coding) video encoding/decoding and playback. For video capture, a component and interface definition for a 6-camera rig and related video capture FPGA cards is developed. Currently, the designed functionality is limited to stationary cameras located in a single-point. For video stitching, a software architecture for merging multiple video feeds into a 360 degrees video sphere is designed by TAU. The implementation of frontend software and hardware components of the real-time 360 degrees video processing pipeline is started. The implemented components include a 3D printed 6-camera rig for six GoPro cameras and related HDMI video capture cards on Pynq FPGAs to enable raw video capture from GoPros. For video stitching, OpenCV stitching algorithm is modified to support 6 input images and optimized for real-time operation. Additionally, to assist CiThruS development, a simulation environment for 360degree traffic imaging is implemented in order to simulate different vehicular camera settings in a virtual city. The simulator is built on top of Unity game engine with assets used from Windridge City and other free sources. The vehicles drive autonomously selecting different routes from a predefined road network and avoid collisions using a simplified collision detections. Pedestrians walk around the city area in defined locations and cross the streets when they have green lights. In addition to TAU's efforts, Nokia, in the first year 
period, specified CiThruS cloud acceleration platform requirements and composed a good understanding of the platform architecture both from the hardware and software perspectives. Also, general purpose cloud acceleration platform descriptions are implemented to further convey CiThruS results to the cloud environment, making it possible to share them with other vehicles in the traffic (Fig. 15).

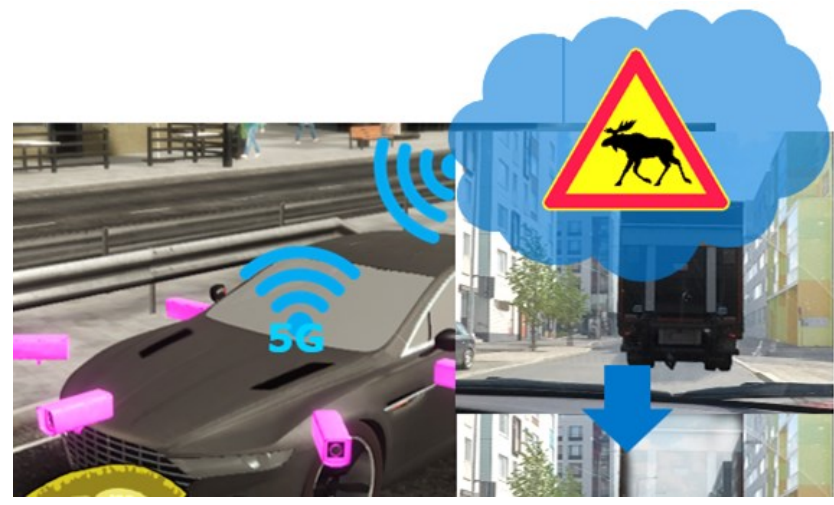

Fig. 15: CiThruS (SuperSight) solution.

e. Control: Sensor fusion capabilities, combined with motion prediction enabled the improvement of AI-based and enhanced controllers that can decide on and implement the required action(s) in the specific situations. Towards this end, a trajectory planning and control algorithm is developed by Polito based on a Model Predictive Control (MPC) approach to work in different road scenarios. The algorithm allows accomplishment of (i) way-point tracking, (ii) lane center tracking, (iii) obstacle avoidance (for fixed and moving obstacles) and (iv) constraint satisfaction (e.g., road boundaries, speed limits, etc.). First year activities of Polito are mainly concerned with the design of a trajectory planning and control algorithm. The MPC trajectory planning and control algorithm is tested in simulation using the Matlab/Simulink simulator. Different road scenarios are considered in these tests, such as avoidance of a fixed obstacle, avoidance of a moving obstacle, way point tracking, lane keeping, lane center tracking, overtaking in a motorway scenario, emergency lane change and stop in a motorway scenario. In these preliminary simulation tests, the MPC algorithm (in all its variants) showed a satisfactory capability of calculating optimal trajectories and provided effective control actions. Two screenshots from two simulations are given in Fig. 16, showing two maneuvers accomplished control in collaboration with CRF and Unimore, using the MPC algorithm for trajectory planning and vehicle control.
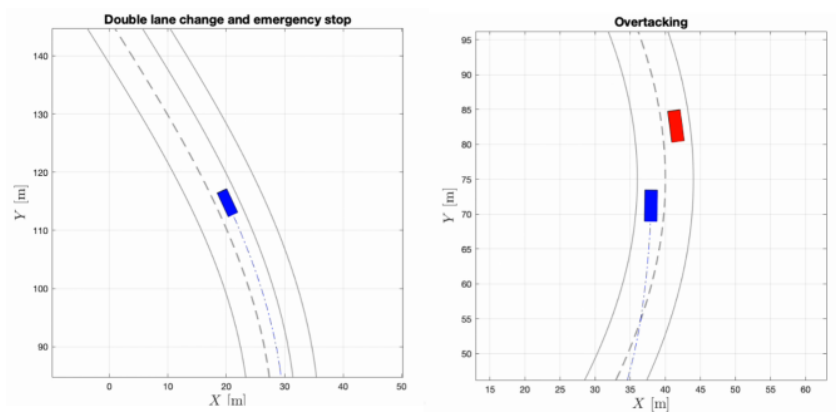

Fig. 16: Two maneuvers accomplished on the simulator using the MPC algorithm for trajectory planning and control.

Towards suspension control, Tenecco evaluated different sensor technologies in the first year for road state scanning. The observed sensor technologies are laser triangulation, Radar and, for a lesser extent, ultrasonic imaging. Laser triangulation is identified as suitable for road scanning, where similar results from LiDAR sensors are expected as soon as they become available from the consortium partners (Innoluce). Radar is also tested for road scanning based on $77 \mathrm{GHz}$ technology, at which Radar becomes usable for road state scanning, as shorter wavelength Radar is still in early design stage. In any case, the required Radar module is a short-range/high bandwidth type to provide the necessary height accuracy, since the specific case of road scanning requires fast repetition rate short-range scanning due to the self-shadowing effect of small objects, especially negative-height objects like potholes. During the first year studies, the smart damper controlling algorithm is implemented in MATLAB/Simulink running on dSpace hardware, where the vehicle controller receives the weighted signals from the physical sensor. In case of vision sensors, each sensor disposes over a dedicated signal pre-processing unit to distribute the processing burden and to preserve bandwidth. For evaluating the performance of the system, 3D street profile data are recorded using a high-resolution laser triangulation system. On these data, different sensor profile simulations can be run and the sensor performance prior to its practical availability can be evaluated. This concerns physical sensors as short-range Radar, LiDAR and vehicle internal readings based on e.g. accelerometers and rattle sensors. The simulated sensor signals are then coupled to a vehicle model in Simulink, where different vehicle suspension models are available. With these models, the sensor performance at nominal conditions and under influence of environmental disturbances are tested

f. Traffic Management: In the transition from $\mathrm{AD}$ levels 2, 3 to level 4 , vehicles need to deal with more complex traffic conditions, road networks, etc. Better understanding of traffic behavior will support the application of traffic measures more adequately, specifically in complex environments such as cities. These new capabilities will support developments of Traffic Management as a Service. The traffic management solution proposed by DAT.Mobility involves fusion of streaming traffic data from traffic controllers, floating car data (FCD) and automatic number-plate recognition (ANPR) cameras. In the first year, a model framework with a network and traffic model is developed (Fig. 17) and a section of Amsterdam city is employed in the model for short term prediction. The efforts of DAT.Mobility will eventually be transferred to SC7 "Shared Control demonstration" (demo\#3) after successful HIL tests finalizing the SC4 studies.

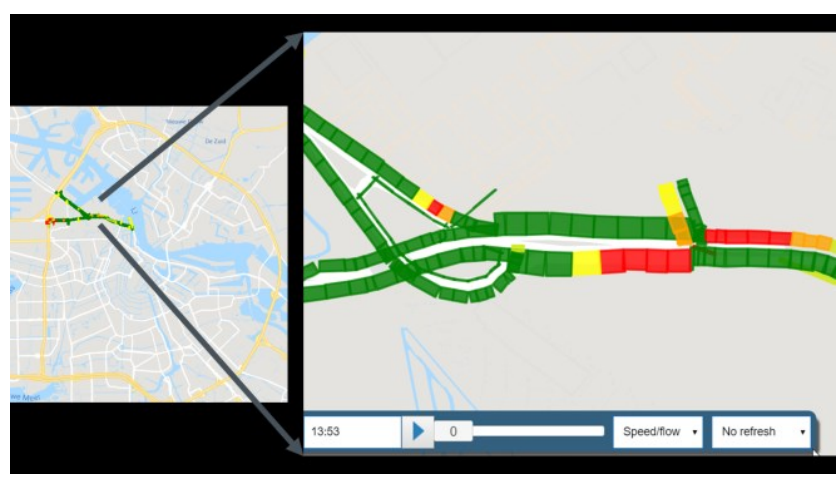

Fig. 17: Framework with network and traffic model for short term prediction.

g. Compute architecture for multi-sensor data fusion: Within the scope of SC4, a compute architecture for multi-sensor data fusion is developed by TUD. In the first year, a simulation framework is set to enable architecture and design space exploration of the compute platform. At the same time, Radar and LiDAR sensor data are obtained to enable the exploration. As part of this effort, creation of a benchmark data set is investigated. A baseline architecture is established for the exploration. Design activities will move towards determining encoding strategies for sensor data in a low-level fusion setup, and subsequently towards system-level design implementation in the second year (Fig. 18). 

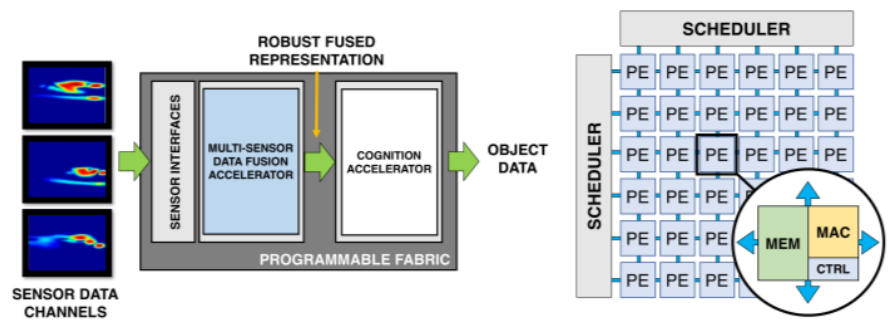

Fig. 18: Conceptual view of TUDelft's accelerator for perception and sensor fusion (left); and an architectural illustration of the massively parallel accelerator (right).

\section{SC5: FUSION application - heavy duty electric vehicle}

SC5 is the first output enabler supply chain that addresses the fourth objective of PRYSTINE:

Objective 4 - Fail-operational systems for urban and rural environments based on FUSION: This objective is achieved by implementing PRYSTINE's fail-operational sensor fusion, content analysis, object recognition, scenario assessment, and decision making solutions on PRYSTINE's fail-operational embedded control and electrical/electronic architectures. Thus, fail-operational automated driving functions for urban and rural environments will be achieved, which will be showcased by PRYSTINE's use-cases.

The vision of SC5 is to integrate the results of supply chains 1-4 in order to achieve the heavy duty vehicle demonstrator employing FUSION, therefore SC5 has close ties with all technology enabler supply chains. The goal is to realize the heavy duty vehicle demonstrator employing fail-operational autonomous driving functions based on FUSION and its data fusion from a wide range of sensors (Radar, LiDAR, camera, ultrasound, etc.) within an integrated platform.

The demonstrator of SC5 will be a Ford heavy duty truck (semi-size trailer). The heavy duty vehicle demonstration will be performed with a Ford F-MAX, introduced by Ford Otosan in 2018. In 2018, Ford F-MAX became available in 29 countries. Ford F-MAX was unveiled during the IAA 2018 in Hannover, Germany and chosen to be the International Truck of the Year 2019.

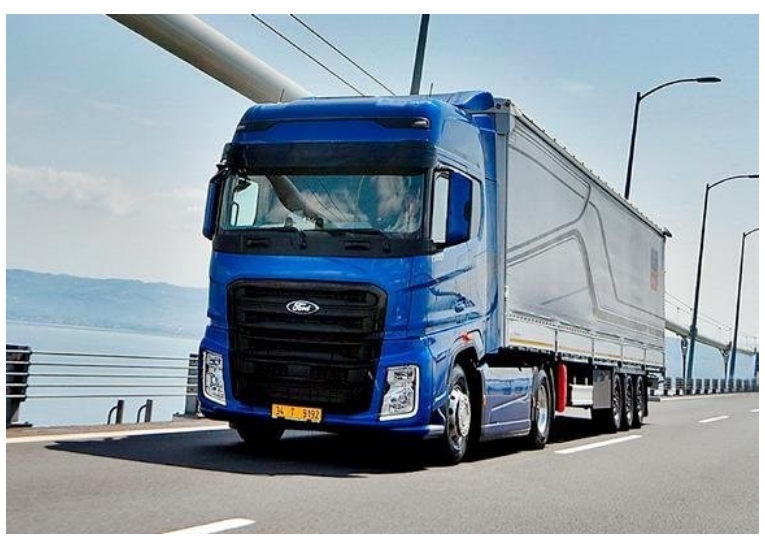

Fig. 19: Ford Otosan's semi-trailer heavy duty vehicle.

Ford F-MAX has a cabin width of 2.5 meters, a 12.7 lt., 500 PS Ecotorq engine, 600 lt. fuel capacity and 3.6 meters axle width. The F-MAX truck is employed as the PRYSTINE heavy duty demonstrator and is equipped with adaptive cruise control, automatic emergency braking and electric steering systems that enable the vehicle to be controlled in lateral and longitudinal axes.

In the context of heavy vehicles, PRYSTINE aims to advance stateof-the-art by realizing an ambitious autonomous heavy-vehicle demonstrator for urban scenarios. Most autonomous developments today concentrate on long-haul highway related topics for heavy- vehicles. The need for high-precision maneuvers, combined with the size of heavy-vehicles, raises a high fatality risk, threatening the accident-free mobility scenarios in urban environments. Towards annihilating these risks, back maneuvering related use cases are defined in the scope of SC5 of PRYSTINE. Specifically, two distinct use cases for trucks with trailers are considered; (i) docking in a docking station and (ii) backing in a construction site. For both use cases, it is common that the driver needs several trials to bring the trailer in the correct position, either to dock correctly to the dedicated slot in the docking station, or to bring the truck in position on the construction site. While the main concern when considering the docking station use case is the time spent to position the trailer, the problem with construction sites is also the surrounding traffic and other road users, such as pedestrians.

These use cases explicitly address the two key performance indicators of SC5; (i) automated back parking of the heavy duty truck at docking station, (ii) automated back entrance of the heavy duty truck into the construction zone.

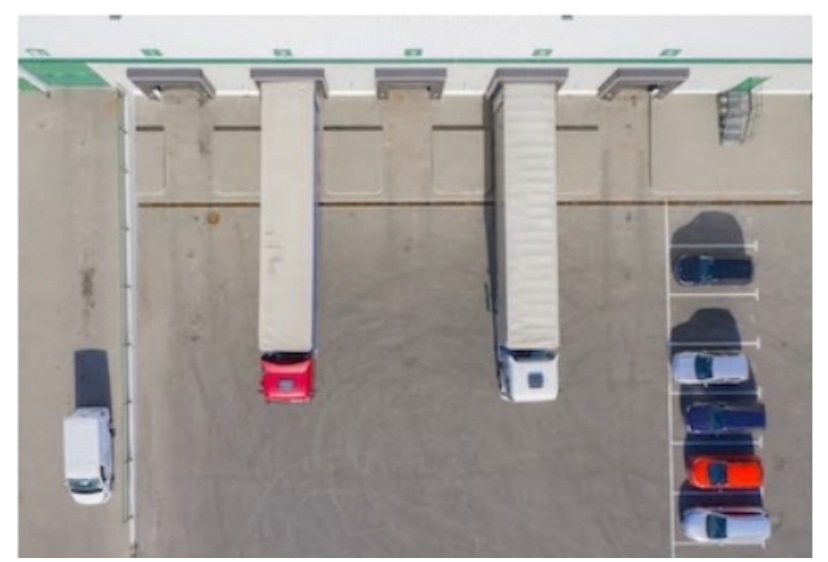

Fig. 20: Use case 1 in SC5: automated back parking of the heavy duty truck at docking station.

There are six partners in SC5 who will contribute to the heavy duty vehicle demonstration; Ford Otosan, AVL-Turkey, Virtual Vehicles, Innoluce, AnyWi and ITI. These contributions take part in both of the use cases defined above and are focused on two scenarios:

a. Traffic Management: Back maneuvering scenario describes the contributions focused on enabling a back maneuvering assist system for heavy duty vehicles.

Ford Otosan will take part in both of the use cases and integrate firstly the sensors; LiDAR, Radar, stereo camera and mono camera to the demo truck. Secondly, Ford Otosan will integrate its perception and fusion algorithms (developed in the scope of SC4) on the target platform, NVidia Drive PX2 that will also be eventually deployed to the demo truck together with a HMI device.

AVL-Turkey will contribute to both of the use cases and integrate its data fusion algorithms developed in the scope of SC4. AVLTurkey will also integrate its trailer-angle detection solution which is crucial in data fusion for mapping local truck and trailer coordinate systems to a reference coordinate system.

Virtual Vehicle will contribute to both of the use cases and integrate its object recognition/classification solution developed in the scope of SC4. Virtual Vehicle will also deploy its solution for failoperationality that will continuously check the status of the algorithms integrated on the demo truck. Like Ford Otosan, Nvidia Drive PX2 will be utilized by Virtual Vehicle as target platform. Thus, the perception outputs of Ford Otosan and Virtual Vehicle will be combined in a single processing unit.

Innoluce will contribute to both of the use cases and integrate up to 5 LiDAR demonstrators with sensor fusion algorithms developed within the scope of SC1 and SC4. 
b. Docking Station Management: Docking station management scenario describes the contributions focused on enabling a facility management system for tracking the status of the docking station by truck drivers and facility managers.

AnyWi will contribute to the use case 1 and integrate its gateway unit developed within the scope of SC3 (see also Fig. 22). According to AnyWi's demonstration scenario, the demo truck will firstly detect docking slot occupancy status and compose docking station suggestion data, which will then be sent to the facility (docking station) management server using the AnyWi Gateway Unit integrated on the truck. A facility management client application (running on a tablet with internet connection) will receive a docking station suggestion from the facility management server and display the docking station suggestion on the HMI to the facility manager. Thus, the facility manager will be able to remotely track docking station occupancy status at the same time.

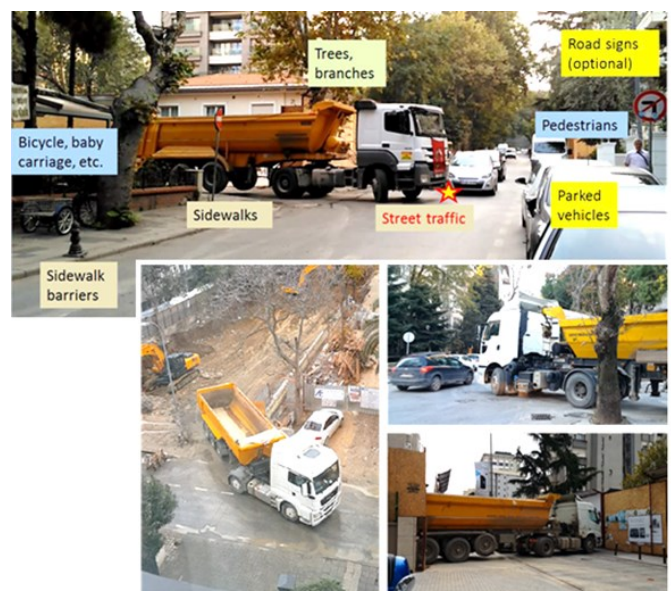

Fig. 21: Use case 2 in SC5: automated back entrance of the heavy duty truck into the construction zone.

ITI will contribute to the use case 1 by integrating its on board unit (OBU) to the demo truck and deploying two road side units (RSU) to the docking station. The road side units will detect several environmental conditions of the station such as temperature, humidity with its embedded sensors and send these data to the demo truck. The on board unit will receive the environmental conditions of the docking station and relay these data to the demo truck, which will eventually display the environmental conditions of the docking station to the driver through the HMI.

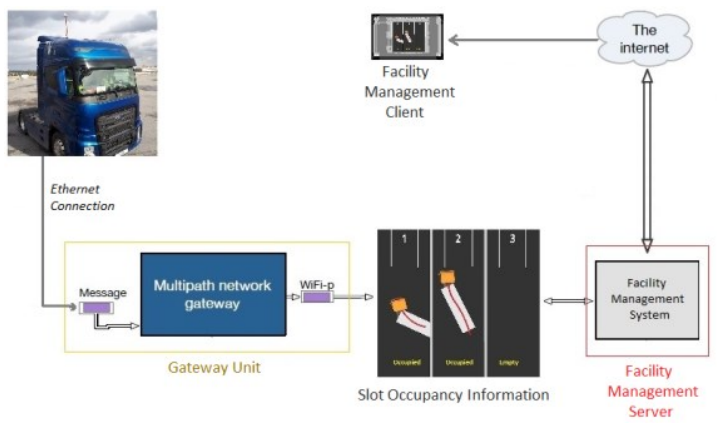

Fig. 22: AnyWi demonstration layout in SC5.

SC6: FUSION application - passenger vehicle

In Supply Chain 6, the FUSION technology developed in the other Supply Chains (SC1-SC3) is integrated in a passenger vehicle demonstrator for test and validation by means of three different use-cases. The demonstrator will be set up in the Modena Automotive Smart Area (MASA), a $4 \mathrm{~km}^{2}$ urban area in the city of
Modena specifically designed for this purpose, which enables the demonstration of the technology in a real urban scenario.

"Fail operational", "sensor fusion strategies", "vehicle-toinfrastructure communication" and "new advanced driver assistance features" are the main aspects that will be theoretically analyzed and then practically implemented and tested among this project.

In the following the description is given of the 3 use-case scenarios of the advanced driver assistant features that will be implemented.

UC6.1 - Traffic light time-to-green. Based on the received traffic light phase schedule, the vehicle calculates the approaching speed at which the vehicle reaches the traffic light, according to the actual traffic condition (vehicles ahead). Fig. 23 depicts this use-case.

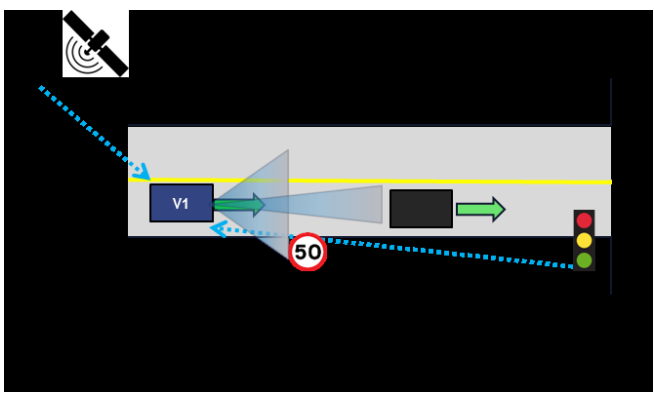

Fig. 23: Description of use-case 6.1.

The information for the development of use-case 1 comes from the following data fusion:

- Maximum allowed speed, based on the fused information coming from the Front Camera (traffic sign recognition), GPS (vehicle location) and the Navigation map (speed limit information)

- Traffic ahead information coming from the Radar (Active Cruise Control).

- Traffic light schedule (time to green/red), via Infrastructure-to-vehicle (I2V) connectivity.

As result of the data fusion of the input data, the output data invehicle is the optimal vehicle speed (slow down/speed up) to reach the green wave (when possible).

UC 6.2 - Vulnerable Road Users (VRU's) and trajectory recognition. The ego-vehicle, with the support of the infrastructure $(\mathrm{I} 2 \mathrm{~V})$, recognizes the type of obstacles in the surroundings of the vehicle and predicts the trajectory of the VRU's (pedestrians and cyclists) in order to avoid potential collisions (see Fig. 24).

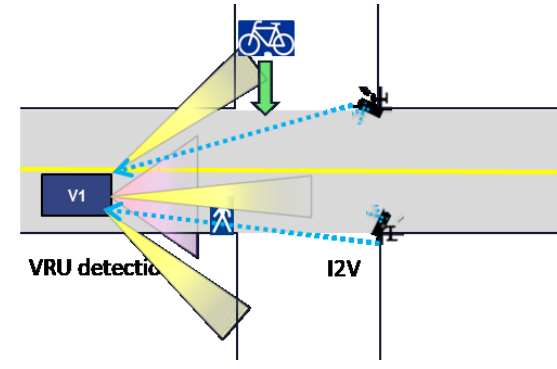

Fig. 24: Description of use-case 6.2.

The input data to carry out this use-case is the following:

- Obstacle detection and classification by on-board cameras.

- Distance and velocity of the obstacles by on-board Radar. 
- Enhanced obstacle identification/classification by onboard LiDAR.

- Information about the type, position and velocity of the occluded obstacles by means of I2V communication.

The output of this use-case is the longitudinal control of the vehicle to avoid potential collision.

UC 6.3 - Driver Monitoring System (DMS) and emergency lateral lane stop. In this use-case a DMS will be implemented, using FUSION components, to detect if the driver is not capable to control the vehicle. In this case, a "take over request" recalls the driver's attention and in case of no driver feedback, a safe stop maneuver is actuated (see Fig. 25).

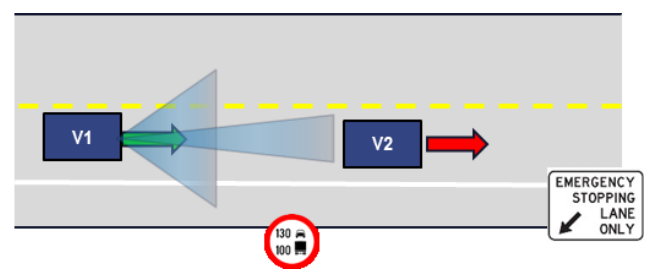

Fig. 25: Description of use-case 6.3

The input information to perform this use-case is hereafter:

- Driver status information (drowsiness, sickness, cognitive load, ecc) based on biometric devices and a dynamic vehicle algorithm that analyzes the driving style.

- Surrounding classification and analysis for a safe stop maneuver, by Radar, cameras, LiDAR and ultrasonic sensors.

The output action in this use-case is the longitudinal/lateral control of the vehicle to avoid potential collision and perform a safe stop maneuver (emergency lights activation and stop in the emergency lane, if possible)

\section{SC7: Shared control and arbitration applications using FUSION}

According to the vision of SC7, the objective is to deploy PRYSTINE's FUSION technologies for the development of an intelligent co-driver able to assist the driver in manual and automated mode for various levels of automation $(2,3$, and 4$)$. The proposed approach for research on AI for the arbitration \& sharing controller elements will include: (i) classification of driver's (and maybe occupants') movement inside the vehicle cockpit; (ii) scenario assessment; (iii) risk assessment; (iv) motion prediction; (v) control sharing and (vi) decision making.

Three distinct demonstrators are developed covering decision and arbitration processes for transitions between human driver and automated system, and between different automation levels all the way up to a fully automated vehicle. Fig. 26 depicts the common framework for the three demonstrators.

Demonstrator 7.1, Driver in the Loop - Shared control and arbitration (simulator), focuses on the study of the interaction between driver and automated system. It implements HiL applications for driver monitoring, recording several variables and using different approaches to establish driver status using FUSION. The simulated vehicle and surrounding variables are also processed and, through FUSION with the driver status, risk assessment is performed. An arbitration algorithm assigns the authority and controls the transitions between the human driver and the automated system. These transitions can be from manual to automated or vice versa and can be initiated either by the driver or by the system.

Use cases are defined to evaluate the arbitration process (i.e. the decision on whether the authority should be given to the driver or the system, and on if/when a transition should take place). Also, the transition itself is a focus for the demonstrator. A smooth dynamic transition, without DDT interruption, warrantying stability and comfort is sought. This transition process is a key factor on the successful implementation of SAE levels 2 to 4 , where the back and forth authority transition between driver and machine is required.

Demonstrator 7.2, Passenger Vehicle and Bus - Layered Control, will put together a fully automated passenger vehicle and an instrumented and connected bus to showcase driver-machine interaction and vehicles cooperation in an urban-like scenario. The automated vehicle will introduce a layered control architecture able to automatically switch from partial to high automation levels, namely SAE Level 2 (Supervised city control); SAE Level 3 (city chauffeur); SAE Level 4 (Safe Stop), following the driving scenario specificities and sensors health. These functionalities will be tested, validated and demonstrated in real conditions.

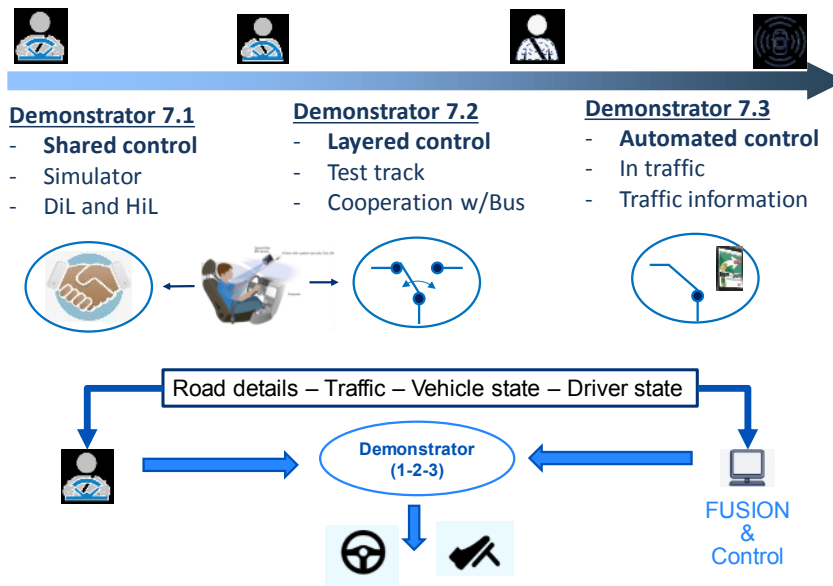

Fig. 26: Common framework of SC7 demonstrators.

With the supervised city control operation, the decision architecture will handle longitudinal and lateral control, and will trigger overtaking when needed, always under the driver supervision. In the city chauffeur mode, the system will take full control in specific complex areas. If a failure is detected, the driver will be requested to intervene, and if no response is obtained, a safe stop will allow stopping the vehicle in a safe manner.

Scene assessment is based on perceiving the outside traffic, driving conditions and the car status along with monitoring the inside car events, (driver's state and behavior). To that end, multiple heterogeneous sensors (GNSS, LiDAR, video, inputs from CAN bus, etc.) will be used. V2X communications will be used to extend the field of perception of each vehicle, so that complex driving scenarios, such as roundabouts or crossroads with occlusions and/or bad visibility can be properly interpreted by the artificial decision system, reducing thus the degree of human intervention. Specific attention will be paid in the use cases to trade control between the driver and the artificial system in any driving scenarios, and more particularly in the transitions between modes. Additionally, the status of the driver in terms of fatigue and distraction will be continuously monitored (i.e. using cameras installed inside the vehicle) to determine the driver readiness to resume the control of the vehicle, and to adapt the HMI communication channel and mechanisms.

Functional validation will be one of the key pillars in this demonstrator, because of its complexity and the introduction of learning and evolving decision systems will require exploring new approaches for fail-operational decision-making systems.

Demonstrator 7.3, Passenger Vehicle - Fully Automated Highway/Urban Decision Making, focuses on the use of AI based algorithms for motion prediction and decision making. The aim is to demonstrate and validate the capability of artificial intelligence methodologies for decision making, in terms of safety, comfort, and possibly throughput. Sensors like LiDAR and Radar will be combined with information on the upcoming traffic state which is communicated (V2I) to the car. The first use case will focus on 
Highway driving, which is more limited in complexity than the second use case of Urban scenarios. Some aspects which can be considered for Highway driving are: lane markings, exits etc. Urban scenarios create much more complexity due to the variety in infrastructure (4 leg crossing, T-shaped crossing, traffic lights) but also for example the variety in road users (pedestrians, different kind of cyclists) or other things to take into account like parked cars, trees and the absence of lane markings.

The car is equipped with a platform for sensor fusion, world modeling and decision making. Although the car will have a driver inside (for safety reasons), the car is considered to be an autonomous driving car (SAE level 4) so the decision-making process in the car does not take the presence of a driver into account. The demonstrator 7.3 will lead to an increase of knowledge about AI for autonomous driving with respect to 'decision making', framework for implementation of AI algorithms, a method to generate/optimize AI algorithms and a first proof-of-concept.

\section{SC8: Novel, competitive and fail-operational semiconductors}

This supply chain will tackle and highlight explicitly the market impact achieved by PRYSTINE's breakthroughs in the field of novel competitive semiconductor systems. Therefore, this supply chain will act as a central platform to showcase novel market figures impacted by PRYSTINE's technology enabling supply chains.

\section{SC9: FUSION's impact on vehicle and road safety}

By consistently enforcing the integration and use of PRYSTINE's novel FUSION technologies throughout the entire project, PRYSTINE will offer an unmatched degree of reliability in the field of autonomous driving. The purpose of this $\mathrm{SC}$ will be to communicate and to showcase (by means of proper demonstrations and methodologies) that road and vehicle safety is impacted in a positive way thanks to PRYSTINE's novel technological advancements.

\section{SC10: End user acceptance of automated driving functions}

Supply Chain 10 (SC10) is the last one, where the FUSION technology developed and integrated by the other Supply Chains (SC1-SC4 and SC5-7, respectively) is evaluated from an end-user's perspective. In particular, with reference to the main goal of PRYSTINE project, the SC10 addresses two specific objectives: O6 "Increased user acceptance of automated driving functions" and O5 "Competitive advantage for European industry". The first includes the following aspects:

- Increasing user acceptance of automated driving functions through PRYSTINE's groundbreaking technological advancements.

- Pivotal impact safety

- Social impact (pivotal impact for mobility of aging population)

The second deals with the impact of PRYSTINE at EU level and is about:

- The increasing market share and revenue of European companies through PRYSTINE's groundbreaking technological advancements (O1-O4).

- $\quad$ The competitive advantage on automotive industry (e.g., marked image of car brands).

- The legislative and regulation impact.

In this paper, we focus specifically on O6, for the evaluation of the demonstrators from the user's acceptance point of view. In particular, the following results are expected:

- Methods and metrics to evaluate the demonstrators as developed in SC5, SC6 and SC7 empirically. This should include a dedicated test-plan (with test cases), including potential real end-users with different degrees of experience with ADAS currently on the market.

- Human participation in these experimental phases (in driving simulator and/or in real cars) to validate the SC57 demonstrators, according to human factors (thus focusing on scenarios where the adaptation between human and machine agents is fundamental for safe and effective operations).

- Measurements of users' expectations and trust in ADFs as developed by PRYSTINE will be provided (using, as baseline, the current available status of automation features).

- Assessment of the effectiveness of adaptation, drivers' situation awareness and trust in automation.

The focus is to prove that the ADF implemented in the demonstrators are well accepted by users (e.g., the co-driver supports human driver in a human-like way and therefore this improves effectiveness and acceptance). Trust in automation before and after driving sessions will be measured, through both questionnaires and implicit measures, such as observation of drivers' behavior during non-drive period. Possible hints of overreliance on automation and misuse / abuse of automation will also be monitored.

This SC considers three demonstrators, above all, the ones developed by the other SCs (SC5, 6 and 7), but evaluated from the end-user perspective. In particular, we will use above all the driving simulator with the participation of naive subjects for safety reason.

This involves the demonstrator 7.1 "Hardware in the Loop Simulator for Shared control and arbitration applications using FUSION" (see the dedicated section for more details).

\section{CONCLUSION}

The automation of vehicles has been identified as one major enabler to master the Grand Societal Challenges 'Individual Mobility' and 'Energy Efficiency'. Highly automated driving functions (ADF) are one major step to be taken. However, at SAE levels, the driver cannot be relied upon to intervene in a timely and appropriate manner, and consequently, the automation must be capable of handling safety-critical situations on its own. For this, failoperational behavior is essential in the sense, plan, and act stages of the automation chain. PRYSTINE's target is to realize Failoperational Urban Surround perceptION (FUSION), which is based on robust Radar and LiDAR sensor fusion, and control functions in order to enable safe automated driving in urban and rural environments.

This work highlights the visions of PRYSTINE's supply chains and summarizes the preliminary results achieved during PRYSTINE's first year. Furthermore, an outlook towards the next years' results is sketched.

\section{ACKNOWLEDGMENT}

The authors would like to thank all national funding authorities and the ECSEL Joint Undertaking, which funded the PRYSTINE project under the grant agreement $n^{\circ} 783190$.

\section{REFERENCES}

[1] N. Druml, G. Macher, M. Stolz, E. Armengaud, D. Watzenig, C. Steger, T. Herndl, A. Eckel, A. Ryabokon, A. Hoess et al., "PRYSTINE Programmable sYSTems for INtelligence in automobilEs," in $201821^{\text {st }}$ Euromicro Conference on Digital System Design (DSD), 2018.

[2] Y. Fu, A. Terechko, T. Bijlsma, P. J. Cuijners, J. Redegeld, and A. O. Örs, "A Retargetable Fault Injection Framework for Safety Validation of Autonomous Vehicles," in IEEE International Conference on Software Architecture Companion (ICSA-C), pp. 69-76, 2019 